\title{
Prevalencia de estereotipias en pacientes sin enfermedades crónicas
}

\author{
ALFREDO EYMANN ${ }^{1}$, MARÍA VERÓNICA LUKICH², MARÍA SOL YOFRE ${ }^{1}$, \\ JULIÁN LLERA ${ }^{1}$, CLARISA MAXIT² \\ 1. Servicio de Clínica Pediátrica, Hospital Italiano de Buenos Aires. \\ 2. Servicio de Neurología Infantil, Hospital Italiano de Buenos Aires.
}

\begin{abstract}
\section{Prevalence of stereotypies in patients without chronic diseases}

Background: Stereotypies have been described in children with developmental disorders but their prevalence and characteristics in developmentally normal children is unknown. Objective: To estimate the prevalence of stereotypies in children without chronic diseases and describe their clinical and epidemiological characteristics. Patients and Methods: A Cross-sectional study was performed. Mothers of children aged from birth to 12 years, who attended the outpatient clinic, answered survey questions. Results: 406 surveys were completed; stereotypies were observed in $57 \%$ of patients, among the most frequent, finger sucking $(21.8 \%)$ and nail biting $(20 \%)$. A multivariate analysis identified that stereotypies are more likely in children with a history of stereotypy in parents, under stressful environments, and with mothers over 35 years old. Conclusion: Stereotypies showed a high prevalence in this study, most frequently in those children under stressful environments, mothers older than 35 years, and affected parents.
\end{abstract}

(Key words: Paroxysms, stereotypies, abnormal movements, developmental disorders).

Rev Chil Pediatr 2013; 84 (3): 262-267

\section{RESUMEN}

Introducción: Las estereotipias han sido descritas en niños con trastornos del desarrollo pero su prevalencia y características en niños con desarrollo madurativo normal no es conocida. Objetivo: Estimar la prevalencia de estereotipias en niños sin enfermedades crónicas y describir sus características clínicas y epidemiológicas Pacientes y Métodos: Estudio de corte transversal. Se aplicó una encuesta a madres de niños recién nacidos hasta niños de 12 años que concurrieron a los consultorios externos con muestreo consecutivo y no aleatorizado. La encuesta fue analizada previamente en un subgrupo de madres. Resultados: Se completaron 406 encuestas, en el $57 \%$ se refirieron estereotipias y las más frecuentes fueron chuparse los dedos $(21,8 \%)$ y comerse las uñas $(20 \%)$. El análisis multivariado identificó mayor probabilidad de presentar estereotipias en niños con antecedentes de esterotipia en los padres, situaciones de estrés y madres mayores de 35 años. Conclusión: Las estereotipias tienen una elevada prevalencia en los niños y son más frecuentes en niños con antecedentes familiares de estereotipias, situaciones de estrés y en niños con madres de mayor edad.

(Palabras clave: Episodios paroxísticos, estereotipias, movimientos anormales, trastornos del desarrollo).

Rev Chil Pediatr 2013; 84 (3): 262-267

Recibido el 15 de febrero de 2012, devuelto para corregir el 11 de junio de 2012, segunda versión el 20 de julio de 2012 , aceptado para publicación el 07 de noviembre de 2012.

Este trabajo cumple con los requisitos sobre consentimiento /asentimiento informado, comité de ética, financiamiento, estudios animales y sobre la ausencia de conflictos de intereses según corresponda.

Correspondencia a:

Alfredo Eymann

E-mail: alfredo.eymann@gmail.com 


\section{Introducción}

Las familias concurren al pediatra para que supervise el crecimiento y desarrollo de sus hijos y poder reflexionar sobre pautas de puericultura y crianza. Es por esto, que el médico puede observar o ser consultado por diferentes manifestaciones conductuales, entre ellas las estereotipias.

Las estereotipias son habituales de observar en niños sanos, han sido definidas como una actividad motora organizada, involuntaria, repetitiva, rítmica, no propositiva de segundos de duración, que conserva siempre un mismo patrón de amplitud y localización ${ }^{1-10}$.

Inicialmente, se dio importancia a la característica rítmica de los movimientos, tanto es así que se las denominaba ritmias motrices ${ }^{2,3}$. En 1994, el DSM IV, las define como "conducta motriz que es repetitiva, a menudo aparentemente orientada y no funcional" $2,3,8,9$.

El año 2001, una revisión de la definición las considera una actividad motriz organizada, repetitiva, no propositiva que es llevada a cabo exactamente de la misma forma en cada repetición, destacando la cualidad de ser una actividad organizada ${ }^{3}$.

En la literatura no médica, la palabra estereotipia se usa como sustantivo para referirse a una "máquina de imprimir en la que se utiliza para cada página una plancha de una pieza..." o para la "repetición involuntaria e intempestiva de un gesto, acción o palabra que ocurre sobre todo en ciertas personas con alteraciones psiquiátricas". O también como adjetivo: "Se aplica al gesto, expresión, actitud, etc, que se adopta formulariamente y no son expresión de un sentimiento afectivo, como por ejemplo, una sonrisa estereotipada" ${ }^{3,11}$.

Las estereotipias pueden ser observadas en niños que padecen un desorden del espectro autista, retraso mental o trastornos de ansiedad, como así también en niños sanos. Los estudios realizados han hecho hincapié en ciertas estereotipias asociadas fundamentalmente a trastornos del desarrollo; por el contrario, las características epidemiológicas y clínicas de estereotipias en niños sanos han sido poco comunicadas.

En este contexto, nos propusimos medir la prevalencia de estereotipias en niños sanos y comparar su frecuencia según edad materna, estrés familiar y antecedentes familiares.

\section{Pacientes y Método}

Estudio de corte transversal. Se invitó a participar a madres de niños recién nacidos hasta 12 años de edad concurrentes a consultorios externos de pediatría por citación programada o demanda espontánea para la atención por pediatría general.

Se excluyeron los niños cuyo acompañante no fuera la madre y/o que presentaran enfermedad crónica o trastornos neurológicos según referencia materna.

Se confeccionó una encuesta diseñada por pediatras y se realizó un ajuste para su comprensión en un grupo de 15 pacientes. No se midió la estructura, ni la validez interna de la encuesta (anexo 1).

Se realizó un muestreo consecutivo y no aleatorizado durante un mes donde la madre de cada niño completó la encuesta en forma de autollenado previo a la consulta y posterior a la aceptación del consentimiento informado oral.

Se definió estereotipias como movimientos repetitivos y rítmicos, no propositivos, que se lleva a cabo exactamente de la misma forma en cada repetición según referencia materna.

Se consideraron las siguientes estereotipias: comer las uñas, morder los labios, chupar algún dedo, golpear la cabeza o el cuerpo, moverse sobre una superficie, tironear el pelo, masturbación frente a otras personas, rechinar los dientes durante el día, morder la mano, protuir la lengua, realizar aleteos manuales y realizar aplausos inmotivados.

Se evaluaron variables demográficas y factores de estrés familiar, definido como: mudanza, cambio de colegio, embarazo o nacimiento de un hermano y/o separación de los padres en el último año (anexo 1).

Las variables continuas se dicotomizaron según su mediana y las categóricas según su frecuencia. Se analizaron las variables categóricas con la prueba de chi cuadrado y se construyó un modelo de regresión logística con las variables biológicas y estadísticamente significativas. Se consideró significativo un valor de 
Tabla 1. Características y prevalencia de estereotipias según sexo y edad

\begin{tabular}{|c|c|c|c|}
\hline Estereotipia & Prevalencia & Edad* & Sexo femenino \\
\hline Chuparse algún dedo & 21,8 & $1 \quad(0,5-3)$ & 56 \\
\hline Comerse las uñas & 20 & $6 \quad(4-10)$ & 56 \\
\hline Morderse los labios & 12 & $6 \quad(4-8,5)$ & 63 \\
\hline Golpear la cabeza o el cuerpo & 10,9 & $1 \quad(0-4,5)$ & 43 \\
\hline Rechinar los dientes & 8,1 & $3 \quad(2-4)$ & 50 \\
\hline Chuparse la mano & 8,1 & $1 \quad(1-2)$ & 37 \\
\hline Aletear las manos & 7,6 & $1 \quad(0-2)$ & 47 \\
\hline Moverse repetitivamente contra alguna superficie & 7 & $1 \quad(1-4)$ & 39 \\
\hline Protruir la lengua & 5,7 & $0 \quad(0-4)$ & 57 \\
\hline Aplaudir & 5,4 & $2(1-4,5)$ & 50 \\
\hline Tirarse el pelo & 3,8 & $1 \quad(1-4)$ & 50 \\
\hline Masturbarse & 2,7 & $2,5(1-5)$ & 40 \\
\hline
\end{tabular}

Número de observaciones $=406$. * Mediana de edad y rango intercuartilo 25-75 en años.

Tabla 2. Variables asociadas para presentar estereotipias

\begin{tabular}{|lcccc|}
\hline & \multicolumn{2}{c}{ Análisis univariado } & \multicolumn{2}{c|}{ Análisis multivariado } \\
\hline Antecedentes en los padres & *Odds Ratio e IC 95\% & valor $\mathbf{p}$ & **Odds Ratio e IC 95\% & valor $\mathbf{p}$ \\
\hline Situaciones de estrés & $8,4[3,4-24,6]$ & $<0,0001$ & $10[4-24]$ & $<0,0001$ \\
\hline Padres mayores de 35 años & $1,8[1,1-2,8]$ & 0,005 & $1,9[1,2-3]$ & 0,006 \\
Tener hermanos & $0,6[0,3-0,9]$ & 0,02 & $0,6[0,4-0,9]$ & 0,04 \\
\hline
\end{tabular}

Número de observaciones $=406 .{ }^{*} \chi{ }^{2}$. ${ }^{*}$ Regresión logística.

p menor a 0,05 . Se utilizó el programa Stata 8.0 (Stata Corporation, Texas, USA). El estudio fue aprobado por el Comité de Ética y Protocolos de Investigación de nuestra institución.

\section{Resultados}

Se entregaron 419 encuestas, de las cuales se completaron 406. Todas las familias tenían seguro de salud y sus necesidades básicas satisfechas, y el $45 \%$ presentaban factores de estrés. La mediana e intervalo intercuartilo 25-75 (IIC) de edad de las madres fue 35 IIC 31- 39 años y el $72 \%$ había finalizado un estudio terciario o universitario.

La mediana e IIC de edad de los niños fue de 4 IIC 2-7 años, el 53\% fueron mujeres y $57 \%$ tenían hermanos.

En el 57\% de los niños encuestados, se re- firieron movimientos compatibles con estereotipias. Las estereotipias más frecuentes fueron chuparse algún dedo $(21,8 \%)$ y comerse las uñas 20\%) (Tabla 1).

El análisis univariado identificó mayor chance de presentar estereotipias con antecedentes en los padres de estereotipias, situaciones de estrés, madres mayores de 35 años y no tener hermanos. El modelo multivariado mantuvo las mismas variables excepto tener hermanos (Tabla 2).

Presentar estereotipias no estuvo asociado al sexo del niño ni a las familias con un sólo adulto conviviente.

\section{Discusión}

La prevalencia de estereotipias fue de un $57 \%$ y, según nuestro conocimiento, este es el 
primer estudio que describe la prevalencia de estereotipias en niños sanos en nuestro medio.

Se considera que las estereotipias pueden ser primarias (presentes en niños sanos) o secundarias (asociadas a una enfermedad neurológica) $)^{1-5,8,9,12}$, tal es así, que se pueden presentar en niños con trastornos del espectro autista, retraso madurativo o retraso mental, trastornos del lenguaje y del aprendizaje ${ }^{1,9,12,13}$.

Las estereotipias varían según la edad siendo distintas las del primer año de vida que las del niño en edad escolar. Es así que en los menores de 3 años las estereotipias más frecuentes fueron golpearse la cabeza, movimiento rítmico sobre una superficie y chuparse algún dedo o tirarse el pelo; en niños en edad escolar lo fueron comerse las uñas y morderse los labios, estos hallazgos coinciden con lo referido en la bibliografia ${ }^{8,9}$.

Un estudio observacional realizado en niños durante su primer año de vida menciona que en 20 niños observados presentaron movimientos rítmicos de miembros superiores, inferiores y golpes contra alguna superficie en todos ellos ${ }^{14}$.

Ante un niño con estereotipias, el pediatra debería considerar varios aspectos: la cualidad (por ejemplo comerse las uñas vs protusión lingual), la edad y el desarrollo del niño. Estos serán los parámetros para diferenciar una estereotipia primaria de una secundaria ${ }^{9}$. Una estereotipia poco prevalente (aletear las manos) debe ser jerarquizada, en cambio, las más frecuentes serán de relevancia cuando se presenten a edades no habituales (chuparse el dedo).

Ante la presencia de conductas motoras se deben también considerar como diagnóstico diferencial a los tics motores complejos, manierismos o disquinesias paroxísticas ${ }^{4,9,10}$.

La presencia de antecedentes familiares fue significativa en nuestro grupo y coincide con lo observado por Harris quien propone un patrón de herencia Mendeliano para las estereotipias motoras con igual distribución por parte de línea paterna o materna ${ }^{1,4,9}$.

El mecanismo fisiopatológico de las este- reotipias tanto primarias como secundarias sigue siendo desconocido tanto es así que se sugieren desde condicionantes ambientales a determinantes neurobiológicos ${ }^{1,4,6,8,9}$.

Al igual que lo referido en la literatura, en nuestro estudio, la aparición o exacerbación de estereotipias se asoció a factores de estrés y, a diferencia de esta, no se observó predominio entre varones y mujeres ${ }^{1,4,15}$. Si bien en otro estudio la prevalencia de estereotipias en diferentes contextos no se modificó, los cambios si estuvieron relacionados con la edad de los niños ${ }^{16}$.

Las estereotipias motoras no suelen traer secuelas físicas ni psicológicas y no requieren tratamiento excepto cuando afectan la calidad de vida de los niños. No existe un tratamiento farmacológico específico, pero, hay experiencias comunicadas con clonazepam, haloperidol, buspirona y naltrexona. Las terapias que tienen como objetivo la modificación de la conducta parecieran ser promisorias ${ }^{1,5,17}$.

Este estudio presenta algunas debilidades. La presencia de estereotipias se evaluó a través de una encuesta a madres y la población representa, en forma mayoritaria, familias urbanas de clase media. Además, es posible que existieran niños con algún trastorno en el desarrollo incluido en la muestra. Por otra parte, este es un estudio exploratorio que sugiere la necesidad de futuras investigaciones para comprender mejor el significado de estas conductas, aunque conocer las características de las estereotipias en niños sin enfermedades crónicas o las más frecuentes por edad le puede permitir al pediatra evitar consultas a los especialistas y una contención familiar más adecuada al considerar a las estereotipias como una expresión del desarrollo normal.

En conclusión, las estereotipias tienen una elevada prevalencia en niños sin enfermedades crónicas y son más frecuentes en presencia de antecedentes familiares de estereotipias, situaciones de estrés y madres de mayor edad. Sin diferencias significativas entre ambos sexos de los niños. 


\section{Anexo 1. Cuestionario para determinar estereotipias}

Esta encuesta tiene como objetivo conocer la frecuencia de la estereotipias en la infancia. Las estereotipias se tratan de movimientos repetitivos y rítmicos, que no tiene una utilidad y que forman parte de la conducta en niños y adultos.

La información es confidencial y será utilizada con fines académicos.

Muchas gracias por su colaboración

Edad del niño/a:

Edad de la madre: años

Sexo del niño/a

femenino

masculino

- Tiene algún hermana/o

si

no

- Señale el número de adultos que conviven en la casa:

- ¿Cuál es la relación habitantes/habitaciones?

- ¿En los últimos 12 meses se separó de su esposo/pareja?

- ¿En los últimos 12 meses se produjo una mudanza?

- ¿En los últimos 12 meses cambió su hijo de escuela?

- ¿En los últimos 12 meses se embarazó o tuvo un hijo?

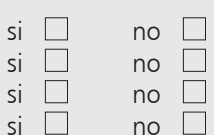

Su hijo/a :

Se come las uñas

Se muerde los labios

Se chupa algún dedo

Tiene movimientos del cuerpo o cabeza repetitivos

Tiene movimientos repetitivos contra alguna superficie

Se tironea el pelo en forma repetitiva

Se masturba frente a las personas

Rechina los dientes durante el día

Se muerde la mano

Protuye su lengua repetidamente

Realiza aleteos manuales

Realiza aplausos inmotivados

Estas conductas se presentan sólo cuando está por dormir o muy cansado

El padre presentó alguna de estas conductas en la infancia

La madre presentó alguna de estas conductas en la infancia

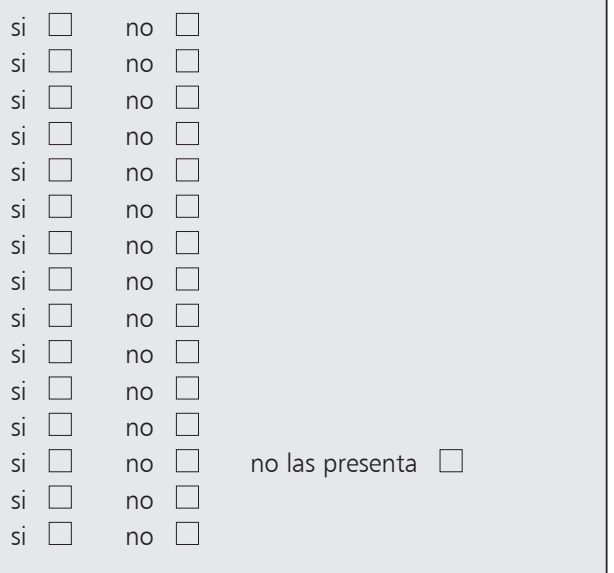

\section{Referencias}

1.- Harris KM, Mahone EM, Singer HS: Nonautistic motor stereotypies: clinical features and longitudinal followup. Pediatr Neurol 2008; 38: 267-72.

2.- Fernández-Álvarez E: Espectro autista. Estereotipias. RevNeurol 2003; 36 (Supl 1): S54-6.

3.- Fernández-Álvarez E: Estereotipias primarias, asociadas y secundarias. Temas de neuropediatria. Homenaje al Dr. Natalio Fejerman 2005; 9: 141-7.

4.- Mahone EM, Dana Brigdes, RN, CFNP, Prahme C, MS, Singer HS, MD: Repetitive arm and hand movements (complex motor stereotypies) in children.The Journal of Pediatrics 2004; 391-5.

5.- Tan A, Salgado M, Stanley Fhan: The characterization and outcome of stereotypic movements in nonautistic children.Movement Disorders 1997; 12: 47-52.
6.- Wolf DS, Singer HS: Pediatric movement disorders: an update. Current Opinion in Neurology 2008; 21: 491-7.

7.- Symons, Sperry, Dropik, Bodfish: The early development of stereotypy and self-injury: a review of research methods. Journal of Intellectual Disability Research 2005; 49: 144-58.

8.- Harvey S, Singer MD: Motor stereotypies. Seminars in Pediatric Neurology 2009; 16: 77-81.

9.- Harvey $S$, Singer MD: Motor stereotypy disorders. Current Opinion in Neurology 2009; 22: 131-6.

10.- Cabrera-López JC, Marti-Herrero M, Fernández-Burriel M, Toledo-Bravo de Laguna L, Domínguez-Ramírez $S$, Fortea-Sevilla $S$ : Síndrome de estereotipias-ticsdistonías paroxísticas. Rev Neurol 2003; 36 (8): 729-34.

11.- Muñoz-Yunta JA, Palau-Baduell M, Díaz F, Aznar G, Veizaga JG, Valls-Santasusana A, et al: Fisiopatogenia de las estereotipias y su relación con los trastornos ge- 
neralizados del desarrollo. Rev Neurol 2005; 41 (supl 1): S139-S47.

12.- Presti MF, Lewis $M H$ : Stratal opioid peptide content in an animal model of spontaneous stereotypic behavior. Behavioural Brain Reserch 2005; 157: 363-8.

13.- Muñoz-Yunta A, Freixas A, Valls-Santasusana A, Maldonado A: Estereotipias, trastornos del desarrollo y estudio con neuroimagen. Revista de Neurología 1999; 28: $124-30$.

14.- Thelen E: Rhythmical stereotipies in normal human infants. Anim Behav 1979; 27: 699-715.
15.- Loh A, Soman T, Brian J, et al: Stereotyped motor behaviors associated with autism in high-risk infants: a pilot videotape analysis of sibiling sample. J Autism Dev Disord 2007; 36: 25-36.

16.- Thelen E: Kicking, rocking and waving: contextual analysis of rhythmical stereotipies in normal human infants. Anim. Behav 1981; 29: 3-11.

17.- Helvink B MD, Holroyd S MD: Buspirone for stereotypic movements in elderly with cognitive impairment. The Journal Neuropsychiatry \& Clinical Neurosciences 2006; 18: 242-4. 